\title{
Influência da modificação da argila montmorilonita nas propriedades mecânicas, termo-mecânicas e morfológicas de nanocompósitos de blendas de poliamida 6/Acrilonitrila-EPDM-estireno
}

\section{Influence of nanoclay modification on the mechanical, thermo-mechanical and morphological properties of polyamide 6/Acrylonitrile-EPDM-Styrene blend-based nanocomposites}

\author{
Amanda Dantas de Oliveira1, Lucas Daniel Chiba de Castro ${ }^{1}$, Marco Keun Jung ${ }^{1}$ e Luiz Antonio Pessan ${ }^{1}$ \\ 'Departamento de Engenharia de Materiais - DEMa, Universidade Federal de São Carlos - UFSCar, \\ São Carlos, SP, Brasil \\ *amandaoliveira82@gmail.com
}

\begin{abstract}
Resumo
O efeito da organofilização da argila no comportamento mecânico, morfológico e termo-mecânico de nanocompósitos baseados em blendas de poliamida 6 )/acrilonitrila-EPDM-estireno foi estudado. Três nanoargilas foram utilizadas para a preparação dos nanocompósitos: duas montmorilonitas comerciais modificadas organicamente (Cloisite ${ }^{\circledR} 30 \mathrm{~B}$ and Cloisite ${ }^{\circledR}$ 20A) e uma montmorilonita sódica não modificada (Cloisite ${ }^{\circledR} \mathrm{Na}^{+}$). Difração de raios-X em alto ângulo, microscopia eletrônica de transmissão e testes de tração e resistência ao impacto foram utilizados para avaliar o efeito da organofilização da nanoargila na morfologia e propriedades mecânicas dos materiais. As propriedades termo-mecânicas foram avaliadas por análise dinâmico mecânica e temperatura de deflexão térmica. Os resultados mostraram que a modificação da nanocarga afeta simultaneamente o grau de reforço da matriz, dispersão das lamelas de argila na blenda e tamanho da fase dispersa AES. A adição das diferentes nanoargilas aumenta o módulo elástico de todos os nanocompósitos ternários em relação à blenda pura sem argila. Por outro lado, um aumento na tenacidade foi obtido apenas para os sistemas com Cloisite ${ }^{\circledR} \mathrm{Na}^{+}$. Um aumento significativo no módulo de armazenamento e HDT foi observado pela incorporação da Cloisite ${ }^{\circledR} 30 \mathrm{~B}$ na blenda.
\end{abstract}

Palavras-chave: organofilização, nanocompósitos, blendas, nanoargilas.

\section{Abstract}

The effect of clay organophilization on mechanical, morphological and thermo-mechanical behavior of polyamide-6/acrylonitrile-EPDM (ethylene/propylene/diene elastomer)-styrene blends was studied. Three nanoclays were used for the preparation of nanocomposites: two commercial organically modified montmorillonites (Cloisite ${ }^{\circledR} 30 \mathrm{~B}$ and Cloisite ${ }^{\circledR} 20 \mathrm{~A}$ ) and an unmodified sodium montmorillonite (Cloisite ${ }^{\circledR} \mathrm{Na}^{+}$). We used wide angle X-ray scattering, transmission electron microscopy and mechanical tests to evaluate the effect of clay modification on the morphology and mechanical properties of the nanocomposites. The thermo-mechanical properties were characterized using dynamic mechanical analysis and heat distortion temperature (HDT). The results show that the modification of clay affected simultaneously the degree of PA6 matrix reinforcement, dispersion of the clay lamellas in the blend and size of the dispersed phase AES. The incorporation of different nanoclays increase the elastic modulus of all the ternary nanocomposites compared to the neat blend. An increase in the toughness is obtained only for systems with Cloisite ${ }^{\circledR} \mathrm{Na}^{+}$. A significant increment in the storages modulus and HDT was observed by the incorporation of Cloisite ${ }^{\circledR} 30 \mathrm{~B}$ in the PA6/AES blends.

Keywords: organophilization, nanocomposites, blends, nanoclays. 


\section{Introdução}

Nos últimos anos, muitas pesquisas têm sido realizadas com o objetivo de desenvolver materiais poliméricos de alto desempenho por meio da nanotecnologia. Uma área de grande importância é a de nanocompósitos poliméricos, onde a matriz é um polímero e cuja fase dispersa (em muitos casos argilas lamelares) possui pelo menos uma de suas dimensões em escala nanométrica ${ }^{[1]}$. A incorporação de $\mathrm{p}$ equenos teores $(\sim 5 \%)$ destas nanocargas ao polímero pode levar a melhorias nas suas propriedades mecânicas, térmicas, de barreira e na inflamabilidade, em magnitude só alcançada quando se utilizam altos teores de cargas tradicionais. $\mathrm{O}$ fator principal para tal desempenho resulta da possibilidade das lamelas de silicatos esfoliarem e se dispersarem individualmente na matriz polimérica e da alta razão de aspecto apresentada por estas cargas ${ }^{[2-4]}$. Adicionalmente, o tratamento orgânico utilizado para tornar a nanoargila mais compatível com o polímero e condições de processamento apresentam grande influência na dispersão das lamelas de silicato e, consequentemente, nas propriedades finais destes materiais ${ }^{[5-7]}$.

As poliamidas são definidas como polímeros pseudo-dúcteis uma vez que apresentam elevada resistência à iniciação da trinca quando comparadas a algumas matrizes frágeis. No entanto, estes polímeros tornam-se frágeis na presença de concentradores de tensão, tais como, um entalhe. Isto é, estes materiais são dúcteis quando não entalhados, mas fraturam de maneira frágil quando entalhados, devido a sua baixa resistência à propagação da trinca ${ }^{[8-10]}$. Para melhorar seu desempenho tem se utilizado a técnica de tenacificação da poliamida com materiais elastoméricos ${ }^{[11-15]}$. Por este método a tenacidade pode ser aumentada, enquanto a resistência à tração e o módulo diminuem proporcionalmente à concentração do elastômero.

$\mathrm{O}$ efeito de argilas modificadas organicamente na morfologia e propriedades de blendas poliméricas tem sido mencionado numerosas vezes na literatura. Estudos com nanocompósitos de poliamida 6 tenacificada com borracha têm mostrado que quando a nanoargila está localizada na fase matriz ocorre uma redução do tamanho dos domínios da fase dispersa ${ }^{[3,4,8,16]}$. Isto muitas vezes foi atribuído ao efeito da nanoargila de prevenir a coalescência dos domínios dispersos e/ou a um efeito compatibilizante apresentado pela nanocarga localizada na interface entre os dois polímeros imiscíveis. Por outro lado, quando a nanoargila está localizada na fase dispersa, frequentemente um aumento do tamanho desta fase é observado.

Dentre os elastômeros disponíveis, o copolímero AES (acrilonitrila-EPDM-estireno) possui grande potencial para a tenacificação de polímeros, tais como a PA6. Características do elastômero EPDM, como, elevada resistência ao impacto, baixa temperatura de transição vítrea, excelente estabilidade térmica e ao ultravioleta, o tornam candidato ideal para a modificação do impacto deste polímero ${ }^{[17]}$. Considerando o efeito da argila de melhorar a desempenho mecânico dos materiais poliméricos, espera-se que a adição de pequenos teores destas nanocargas às blendas de PA6/AES, melhore as propriedades mecânicas e termo-mecânicas destes materiais sem comprometer a sua processabilidade.

Este trabalho tem como objetivo estudar o efeito da modificação da argila montmorilonita no comportamento mecânico, morfológico e termo-mecânico de nanocompósitos baseados em blendas de PA6/AES. Para este propósito, diferentes argilas montmorilonitas foram utilizadas para a preparação dos nanocompósitos poliméricos com o intuito de avaliar o efeito de nanoreforço bem como o efeito compatibilizante apresentando por estas nanocargas.

\section{Experimental}

\subsection{Materiais}

A poliamida 6 de especificação B300 foi fornecida pela Polyform, possui índice de fluidez de $2,9 \mathrm{~g} / 10 \mathrm{~min}\left(235^{\circ} \mathrm{C} / 2,16 \mathrm{Kg}\right)$ e densidade de $1,13 \mathrm{~g} / \mathrm{cm}^{3}$. O acrilonitrila-EPDM-estireno (AES) utilizado foi o Royaltuf 372P20, fornecido pela Chemtura Corporation. Este material é constituído de EPDM, SAN e EPDM graftizado com SAN (EPDM-g-SAN). Esta resina contém 50\% de EPDM e 50\% de SAN (valores fornecidos pelo fabricante) e $27 \%$ de acrilonitrila no SAN livre ${ }^{[18]}$. Para a obtenção dos nanocompósitos foram utilizadas três montmorilonitas comerciais: Cloisite ${ }^{\circledR} 30 \mathrm{~B}$, Cloisite ${ }^{\circledR} 20 \mathrm{~A}$ e Cloisite ${ }^{\circledR} \mathrm{Na}^{+}$. As argilas foram fornecidas pela Southern Clay Products Inc. A Cloisite ${ }^{\circledR} 30 \mathrm{~B}$ é uma montmorilonita natural quimicamente modificada com um sal quaternário de amônio que possui grupamentos polares, possui densidade aparente de aproximadamente $0,36 \mathrm{~g} / \mathrm{cm}^{3}$, perda de massa durante a queima de $30 \%$ e espaçamento basal de $1,85 \mathrm{~nm}$. A Cloisite ${ }^{\circledR} 20$ A é uma montmorilonita modificada organicamente com um sal de amônio quaternário apolar, possui densidade aparente de aproximadamente $0,32 \mathrm{~g} / \mathrm{cm}^{3}$, perda de massa durante a queima de $38 \%$ e espaçamento basal de 2,32 nm. Já a Cloisite ${ }^{\circledR} \mathrm{Na}^{+}$é uma montmorilonita sódica natural e possui densidade aparente de $0,33 / \mathrm{cm}^{3} \mathrm{e}$ espaçamento basal inicial de $1,17 \mathrm{~nm}$.

\subsection{Preparação dos nanocompósitos}

A blenda PA6/AES e os nanocompósitos foram preparados a partir da mistura no estado fundido em uma extrusora dupla rosca co-rotacional MT19TC da marca B\&P Process Equipment Systems com diâmetro de $19 \mathrm{~mm}$ e $\mathrm{L} / \mathrm{D}=25$ com velocidade das roscas de $160 \mathrm{rpm}$ e com um perfil de temperatura de $200^{\circ} \mathrm{C} / 220^{\circ} \mathrm{C} / 220^{\circ} \mathrm{C} / 220^{\circ} / 230^{\circ} \mathrm{C}$.

Para a obtenção dos nanocompósitos de blenda PA6/AES e nanoargilas, os componentes foram misturados manualmente e alimentados simultaneamente na extrusora. Antes da preparação das misturas, a PA6 e o AES foram pulverizados com o auxílio de um moinho criogênico (Modelo Mikro-Bantam, da Micron Powder Systems) e, em seguida, submetidos à secagem em estufa a vácuo a temperatura de $80^{\circ} \mathrm{C}$, por um período de 24 horas.

Os nanocompósitos poliméricos foram preparados como misturas binárias de PA6/argila $(95 / 5 \%)$ e ternárias de PA6/AES/argila (66,5/28,5/5\%). Os nanocompósitos binários foram designados por: PA6/30B, PA6/20A e PA6/Na e os sistemas ternários como: $\mathrm{PA} 6 / \mathrm{AES} / 30 \mathrm{~B}$ - nanocompósitos de blendas de PA6/AES com a Cloisite ${ }^{\circledR} 30 \mathrm{~B}$; PA6/AES/20A - nanocompósitos de blendas de PA6/AES com a Cloisite ${ }^{\circledR} 20 \mathrm{~A}$ e PA6/AES/Na - nanocompósitos de blendas de PA6/AES com a Cloisite ${ }^{\circledR} \mathrm{Na}^{+}$. Os materiais puros e a blenda PA6/AES (70/30\%) também foram preparados nas mesmas condições e seus resultados foram utilizados para efeitos comparativos. 


\subsection{Moldagem dos corpos de prova por injeção}

Após todas as amostras obtidas por extrusão terem sido granuladas e secadas, elas foram submetidas ao processo de moldagem por injeção em injetora da marca Arburg Allrounder, modelo $270 \mathrm{~V}$, com perfil de temperatura de $230{ }^{\circ} \mathrm{C} / 240{ }^{\circ} \mathrm{C} / 240{ }^{\circ} \mathrm{C} / 240{ }^{\circ} \mathrm{C} / 245{ }^{\circ} \mathrm{C}$. Outras condições utilizadas foram velocidade da rosca de $12 \mathrm{~m} / \mathrm{min}$, temperatura do molde a $50^{\circ} \mathrm{C}$ e tempo de resfriamento 30 segundos.

\subsection{Caracterização estrutural}

O grau de intercalação/esfoliação da nanoargila nos nanocompósitos foi avaliado através de análises de difração de Raios-X de alto ângulo (WAXD) e microscopia eletrônica de transmissão (MET).

As análises de WAXD foram realizadas utilizando-se se um difratômetro Rigaku, modelo Ultima IV, com radiação de $\mathrm{CuK}_{\alpha}(\lambda=1,542 \AA)$, operando a $40 \mathrm{KV}$ e $40 \mathrm{~mA}$. A varredura foi realizada na faixa de $2 \theta$ entre $1,5^{\circ}$ e $10^{\circ}$, a uma taxa de $1 \%$ min

A dispersão das partículas de AES nos nanocompósitos, bem como o estado de dispersão das argilas na matriz e na blenda de PA6/AES, foram estudados através de microscopia eletrônica de transmissão (MET), utilizando-se um microscópio Philips, modelo CM120, operando a 120KV.

As amostras da blenda (PA6/AES) e dos nanocompósitos analisadas foram retiradas de corpos de prova de impacto do tipo Izod injetados. As amostras foram retiradas da região média do comprimento do corpo de prova, na direção perpendicular ao fluxo de injeção. Em seguida essas amostras foram submetidas ao "trimming" (corte na forma de trapézio) e foram criogenicamente microtomadas, ou seja, cortadas em seções ultrafinas, $40 \mathrm{~nm}$ de espessura, com facas de diamante, por um micrótomo tipo Riechert-Jung Ultracut E, com temperatura da amostra entre -60 e $-75^{\circ} \mathrm{C}$ e temperatura da faca em $-50^{\circ} \mathrm{C}$. Foram utilizadas telas ou "grids" de cobre para coletar as amostras fatiadas que se encontravam imersas em uma solução de DMSO:água (3:2). Após secagem, as amostras foram tingidas com vapor de solução de tetróxido de rutênio $\left(\mathrm{RuO}_{4}\right)$ por 2 horas. Este reagente ancora preferencialmente os anéis aromáticos das unidades monoméricas de estireno do copolímero SAN presente no AES, de modo que a fase escura observada nas micrografias pode ser atribuída à fase SAN. Após o tingimento, os "grids" com as amostras tingidas foram analisados no microscópio eletrônico de transmissão.

\subsection{Caracterização mecânica}

Os ensaios de resistência mecânica sob tração foram realizados segundo a norma ASTM D638, utilizando uma máquina universal Instron, modelo 5569, sendo utilizada uma célula de carga de $50 \mathrm{KN}$, velocidade de $5 \mathrm{~mm} / \mathrm{min}$ com a utilização de um extensômetro.

A resistência ao impacto foi medida em corpos de prova com entalhe utilizando um equipamento de impacto tipo pêndulo Ceast, modelo RESIL 25, acoplado a um sistema de aquisição de dados DAS 4000. Oito amostras foram testadas para cada composição, e foi utilizado um martelo de 2,75 J. Os corpos de prova obtidos por moldagem por injeção foram entalhados em uma máquina de entalhe marca CEAST, com profundidade de 2,54 $\pm 0,1 \mathrm{~mm}$, com velocidade mínima de entalhe e segundo a norma ASTM D256, verificando-se a profundidade do entalhe em um micrômetro apropriado.

\subsection{Análise Dinâmico-Mecânica (DMA)}

A análise dinâmico-mecânica foi realizada em um equipamento DMA da TA Instruments, modelo Q800. O modo de solicitação dinâmico-mecânica utilizada foi o de flexão em dois pontos. A taxa de aquecimento utilizada foi de $2^{\circ} \mathrm{C} / \mathrm{min}$ e a frequência foi de $1 \mathrm{~Hz}$. Intervalo de temperatura utilizado foi de $-100^{\circ} \mathrm{C}$ a $140^{\circ} \mathrm{C}$.

\subsection{Temperatura de Deflexão Térmica (HDT)}

$\mathrm{O}$ ensaio de HDT foi realizado em um equipamento da CEAST, modelo HDT 6 VICAT, P/N 6921.000 de acordo com a norma ASTM D 648, com taxa de aquecimento de $120^{\circ} \mathrm{C} / \mathrm{h}$, até que o defletisse $0,25 \mathrm{~mm}$ sob uma tensão de $1800 \mathrm{kPa}$.

\section{Resultados de Discussão}

\subsection{Caracterização estrutural}

A Figura 1 apresenta os resultados das análises por WAXD das nanoargilas, dos sistemas binários de PA6 e AES com as diferentes nanoargilas e dos sistemas ternários (PA6/AES/argila). Os valores dos ângulos de difração e dos espaçamentos basais das montmorilonitas (puras e nos nanocompósitos) estão apresentados na Tabela 1 .

Observa-se na Figura 1a que o nanocompósito binário PA6/30B não apresenta pico de difração, isto sugere que as

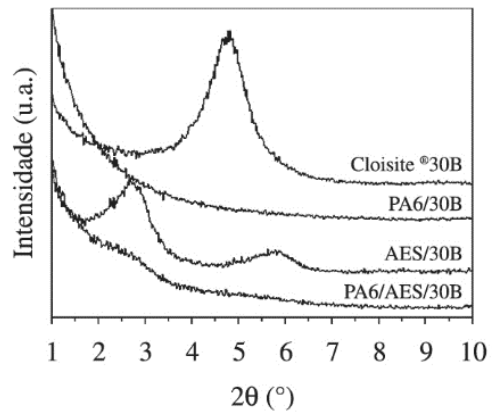

(a)

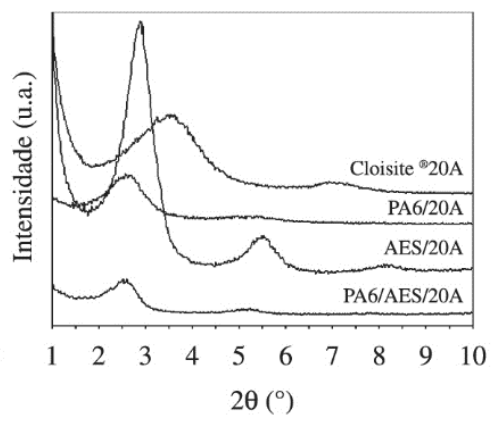

(b)

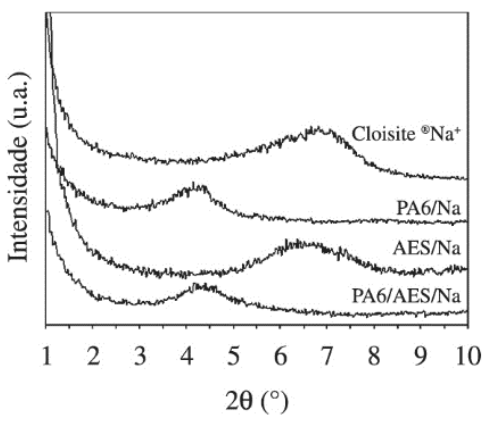

(c)

Figura 1. Difratogramas de raios-X das argilas puras, dos nanocompósitos binários (PA6/argila e AES/argila) e sistemas ternários (PA6/ AES/argila): (a) sistemas com Cloisite ${ }^{\circledR 0 B}$; (b) sistemas com Cloisite ${ }^{\circledR} 20 \mathrm{~A}$; (c) sistemas com Cloisite ${ }^{\circledR} \mathrm{Na}^{+}$. 
Tabela 1. Valores de distâncias basais das montmorilonitas, calculados a partir dos difratogramas de WAXD.

\begin{tabular}{|c|c|c|}
\hline Amostra & $2 \theta\left({ }^{\circ}\right)$ & $d_{001}(\mathrm{~nm})$ \\
\hline Cloisite $^{\circledR 30 B}$ & 4,8 & 1,83 \\
\hline PA6/30B & --- & ----- \\
\hline $\mathrm{AES} / 30 \mathrm{~B}$ & 2,70 & 3,26 \\
\hline PA6/AES/30B & 2,60 & 3,39 \\
\hline Cloisite $^{\circledR} 20 \mathrm{~A}$ & 3,53 & 2,50 \\
\hline PA6/20A & 2,60 & 3,39 \\
\hline $\mathrm{AES} / 20 \mathrm{~A}$ & 2,88 & 3,06 \\
\hline PA6/AES/20A & 2,55 & 3,47 \\
\hline Cloisite ${ }^{\circledR} \mathrm{Na}^{+}$ & 6,91 & 1,27 \\
\hline $\mathrm{PA} 6 / \mathrm{Na}$ & 4,17 & 2,12 \\
\hline $\mathrm{AES} / \mathrm{Na}$ & 6,67 & 1,32 \\
\hline PA6/AES/Na & 4,34 & 2,03 \\
\hline
\end{tabular}

lamelas de argila encontram-se completamente esfoliadas nestes sistemas. Por outro lado, o nanocompósito AES/30B apresentou um pico de difração com distância de $\mathrm{d}_{001}=1,63 \mathrm{~nm}$, indicando a formação de estrutura intercalada. Para o sistema ternário PA6/AES/30B, verificou-se a presença de um ombro em aproximadamente $2 \theta=2,6^{\circ}$, correspondendo a um espaçamento $d_{001}$ de $3,39 \mathrm{~nm}$, sugerindo um processo de esfoliação parcial.

Para os nanocompósitos obtidos a partir da Cloisite ${ }^{\circledR} 20 \mathrm{~A}$ (Figura 1b), o deslocamento dos picos de difração para ângulos menores indica que durante o processamento houve intercalação das cadeias poliméricas entre as camadas de argila. Para estes sistemas, a esfoliação das lamelas de argila não foi observada, provavelmente devido às baixas interações entre as cadeias de ambos os polímeros com a Cloisite ${ }^{\circledR} 20$ A. O maior valor de espaçamento basal encontrado para os nanocompósitos de PA6 (3,39 nm) em relação aos nanocompósitos de AES (3,06 nm) sugere uma maior intercalação de PA6; isto pode ser resultado de uma melhor interação da PA6, em comparação com o AES, com as lamelas da nanoargila. Além do plano (l0 0 1), pode-se observar um segundo ombro $\left(2 \theta=7,0^{\circ}\right)$ para a nanoargila que pode ser atribuído a distância $\mathrm{d}_{002}$ que é um plano paralelo ao $\mathrm{d}_{001}{ }^{[19]}$. Para os nanocompósitos esse ombro aparece apenas nos sistemas AES/20A.

Já para os nanocompósitos obtidos comaCloisite ${ }^{\circledR} \mathrm{Na}^{+}$(Figura 1c), foram verificados maiores aumentos nos valores $\mathrm{de}_{001}$ para os sistemas PA6/Na e PA6/AES/Na, indicando aumento da distância basal da argila, sugerindo a formação de estrutura intercalada para estes sistemas. O nanocompósito binário AES/Na não apresentou variações significativas no valor de $\mathrm{d}_{001}$ em relação à Cloisite ${ }^{\circledR} \mathrm{Na}^{+}$pura. Observa-se ainda nesta figura, que o sistema PA6/AES/Na possui valor de $d_{001}$ similar ao nanocompósito binário PA6/Na. Isto sugere que para a mistura ternária a argila Cloisite ${ }^{\circledR} \mathrm{Na}^{+}$tende a residir preferencialmente na fase matriz, embora não é completamente excluída a possibilidade que uma pequena quantidade argila intercalada possa existir na fase dispersa AES.

\subsubsection{Dispersão da nanoargila}

As micrografias obtidas por microscopia eletrônica de transmissão dos nanocompósitos binários de PA6/argila estão apresentadas na Figura 2.

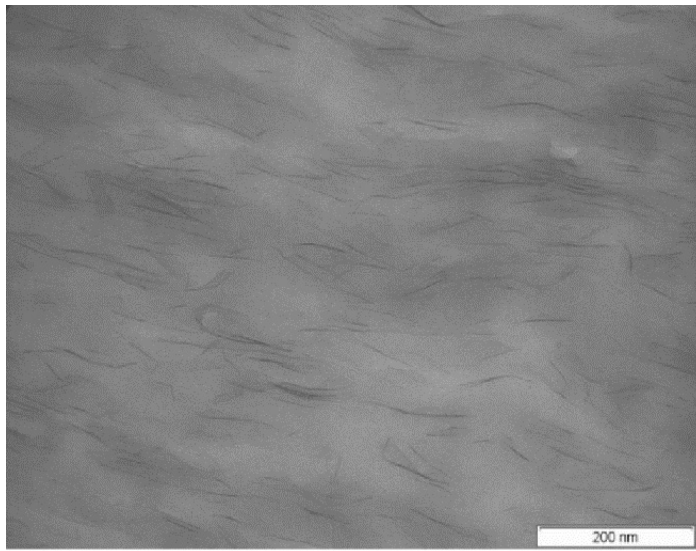

(a)

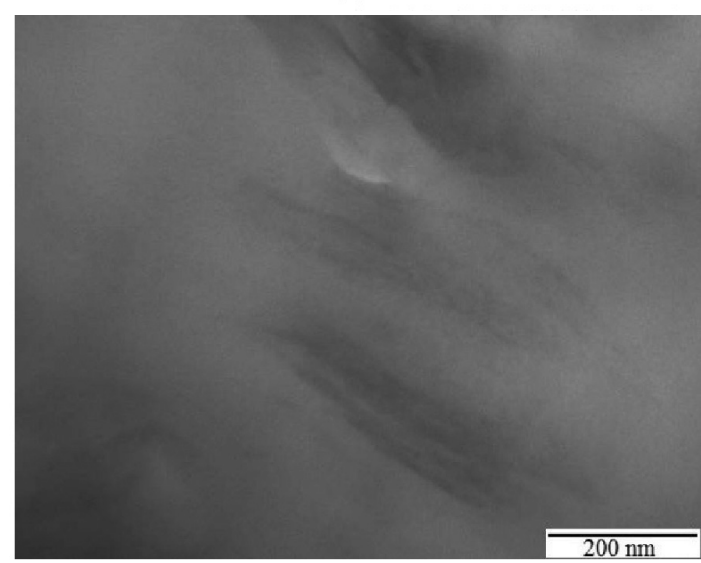

(b)

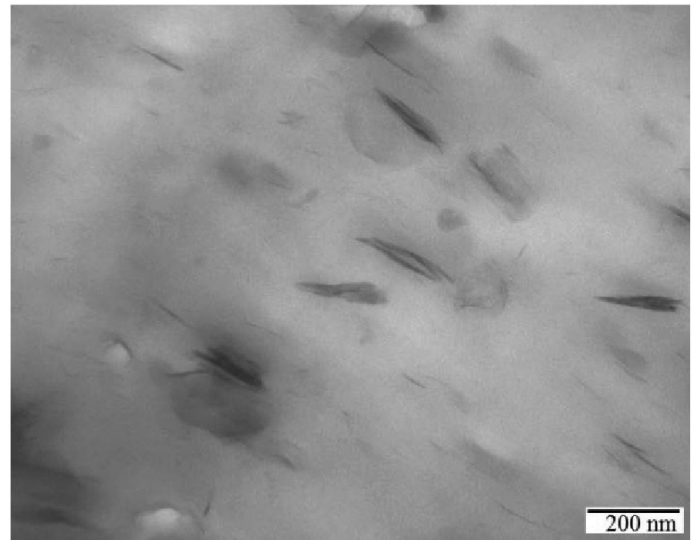

(c)

Figura 2. Micrografias obtidas por MET dos nanocompósitos binários: (a) PA6/30B (110.000x); (b) PA6/20A (110.000x) e (c) PA6/Na (66.000x).

Como esperado, o nanocompósito PA6/30B exibe uma morfologia bem esfoliada consistindo de uma predominância de partículas de argila dispersas individualmente na matriz polimérica. Na Figura 2b, nanocompósito PA6/20A, observam-se estruturas intercaladas. Por fim, para o nanocompósito PA6/Na (Figura 2c) observa-se a presença de pequenos tactóides de argila distribuídos pela matriz. 
A Figura 3 apresenta as micrografias obtidas por MET dos sistemas ternários com diferentes nanocargas.

As imagens obtidas para estes nanocompósitos corroboram com os resultados de WAXD desses sistemas: observa-se uma estrutura esfoliada para PA6/AES/30B, estrutura intercalada para PA6/AES/20A (Figura 3b) e, finalmente, a presença de tactóides de argila montmorilonita nos sistemas ternários de PA6/AES/Na.

Pode-se observar também que as lamelas de argila nesses nanocompósitos estão localizadas na fase matriz, mas algumas partículas de argila migram para a interface PA6/AES e tendem a envolver as partículas de AES. Isto foi particularmente observado para a mistura PA6/AES/30B. Verifica-se ainda que para os três sistemas estudados aparentemente as lamelas de argila não estão presentes na fase dispersa AES.

Uma avaliação dos resultados de WAXD e MET para os nanocompósitos obtidos com diferentes nanoargilas mostra o efeito da estrutura das nanocargas na morfologia destes sistemas. Como esperado, a Cloisite ${ }^{\circledR}$ 30B por ser modificada com um surfactante polar apresenta melhor interação com polímeros polares, tais como, a PA6. Esta forte interação é responsável pela melhor dispersão da argila para estes nanocompósitos em relação aos sistemas com Cloisite ${ }^{\circledR} 20 \mathrm{~A}$ e Cloisite ${ }^{\circledR} \mathrm{Na}^{+}$. Por outro lado, a interação entre as cadeias da PA6 com a Cloisite ${ }^{\circledR} 20$ A é menor, uma vez que esta nanocargas é tratada com surfactante apolar. Por fim, a presença de tactóidos nos sistemas com Cloisite ${ }^{\circledR} \mathrm{Na}^{+}$é resultado de baixas interações entre a matriz com Cloisite ${ }^{\circledR} \mathrm{Na}^{+}$sem tratamento.

\subsubsection{Dispersão da Fase AES}

O efeito da organofilização da argila na dispersão da fase AES também foi estudado e os resultados são mostrados na Figura 4. As amostras foram tingidas com $\mathrm{RuO}_{4}$, o que leva a observação de uma fase escura que corresponde ao SAN, que parece estar disperso de modo heterogêneo em uma matriz de EPDM, e distinguir também a fase PA6 (regiões mais claras) da fase AES.

Para a blenda pura sem argila (Figura 4a), observa-se uma morfologia constituída de partículas grosseiras de AES dispersas de forma não uniforme e com heterogeneidade de tamanho de partículas, o que se correlaciona com as baixas propriedades mecânicas. Com a incorporação de 5\% em massa de nanoargila Cloisite ${ }^{\circledR}$ 30B (Figura 4b), ocorre uma redução do tamanho desses domínios e eles tendem a ficar mais alongados e dispersos. A presença das lamelas de silicato na matriz polimérica aumenta o

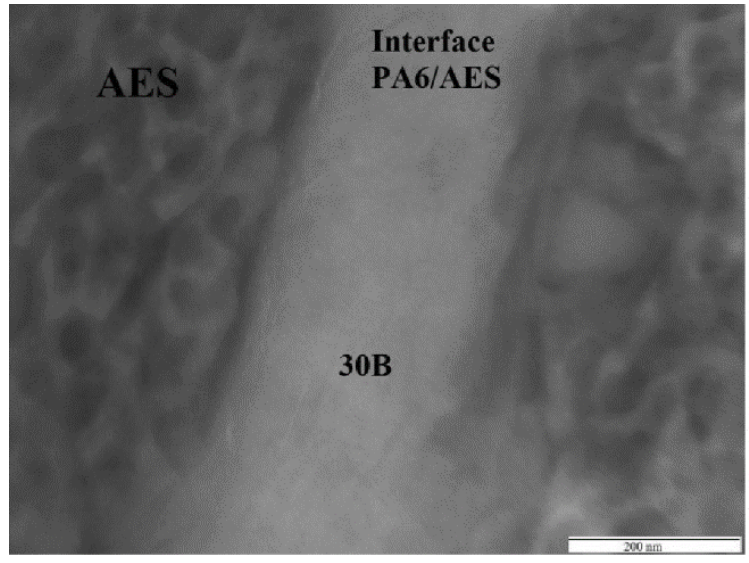

(a)

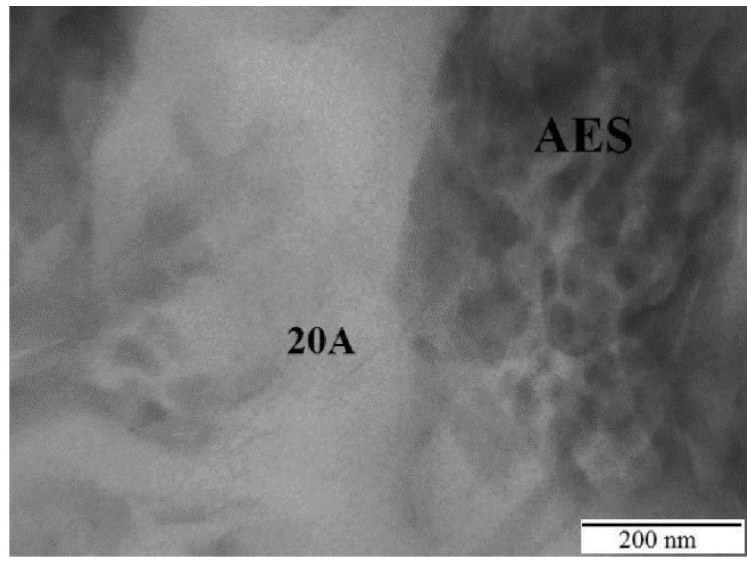

(b)

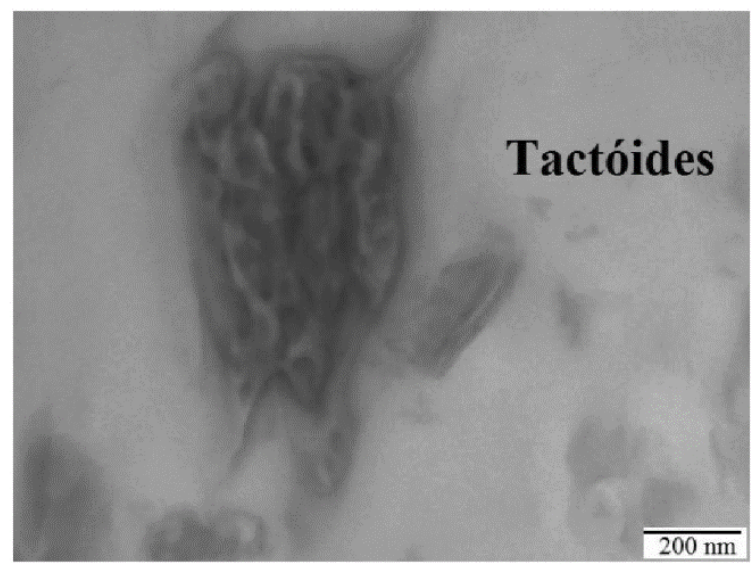

(c)

Figura 3. Micrografias obtidas por MET dos nanocompósitos ternários: (a) PA6/AES/30B; (b) PA6/AES/20A e (c) PA6/AES/Na. Aumento de 110.000x. 
peso molecular desta fase e consequentemente a sua viscosidade; isto é acompanhado por uma redução do tamanho de partículas da fase dispersa ${ }^{[4]}$. Para o sistema ternário com Cloisite ${ }^{\circledR} 20 \mathrm{~A}$ são observados grandes aglomerados de borracha. Por fim, analisando a morfologia apresentada pelo sistema PA6/AES/Na, verifica-se que os domínios de AES apresentam-se alongados e com tendência a co-continuidade.

\subsection{Caracterização mecânica}

A Tabela 2 apresenta os resultados obtidos através de ensaios de tração uniaxial para os polímeros puros, para a blenda binária, para os nanocompósitos binários e sistemas ternários.
Como observado os nanocompósitos binários (PA6/argila) apresentaram um aumento no valor do módulo em relação a PA6 pura. Por outro lado, a incorporação das nanocargas à matriz polimérica diminui drasticamente $\mathrm{a}$ deformação na ruptura. Trabalhos anteriores mostram que quanto maior a dispersão da argila e maior o grau de intercalação/esfoliação, maior será a área superficial da mesma e com isso maior o efeito de reforço da matriz pela argila. Dos vários parâmetros obtidos dos ensaios mecânicos, o módulo elástico é o melhor indicador do grau de esfoliação das camadas da argila ${ }^{[3,20]}$. Para os sistemas binários apresentados na Tabela $2 \mathrm{o}$ aumento do módulo é mais pronunciado no nanocompósito com Cloisite ${ }^{\circledR} 30 \mathrm{~B}$, o qual apresenta estrutura esfoliada, enquanto

Tabela 2. Propriedades mecânicas dos sistemas estudados

\begin{tabular}{|c|c|c|c|c|}
\hline Material & $\begin{array}{c}\text { Módulo de Elasticidade } \\
\text { (GPa) }\end{array}$ & $\begin{array}{c}\text { Tensão no Escoamento } \\
\text { (MPa) }\end{array}$ & $\begin{array}{c}\text { Deformação na ruptura } \\
(\%)\end{array}$ & $\begin{array}{c}\text { Resistência ao impacto } \\
(\mathrm{J} / \mathrm{m})\end{array}$ \\
\hline PA6 & $3,2 \pm 0,05$ & $76,6 \pm 2,5$ & $34,5 \pm 6,8$ & $35,5 \pm 3,0$ \\
\hline PA6/AES & $1,8 \pm 0,08$ & $35,0 \pm 0,5$ & $38,5 \pm 6,5$ & $64,4 \pm 4,0$ \\
\hline PA6/30B & $4,2 \pm 0,10$ & $80,7 \pm 1,7$ & $6,0 \pm 0,4$ & $51,0 \pm 2,0$ \\
\hline PA6/AES/30B & $3,2 \pm 0,09$ & $44,0 \pm 1,1$ & $1,7 \pm 0,1$ & $35,7 \pm 1,2$ \\
\hline PA6/20A & $3,5 \pm 0,06$ & $64,8 \pm 1,0$ & $10,0 \pm 2,2$ & $54,0 \pm 1,7$ \\
\hline PA6/AES/20A & $2,1 \pm 0,03$ & $32,6 \pm 0,6$ & $7,0 \pm 0,4$ & $38,4 \pm 3,0$ \\
\hline $\mathrm{PA} 6 / \mathrm{Na}$ & $3,3 \pm 0,07$ & $72,5 \pm 1,2$ & $21,5 \pm 2,1$ & $49,8 \pm 2,0$ \\
\hline PA6/AES/Na & $2,1 \pm 0,03$ & $38,1 \pm 0,4$ & $18,0 \pm 1,7$ & $73,8 \pm 4,3$ \\
\hline
\end{tabular}

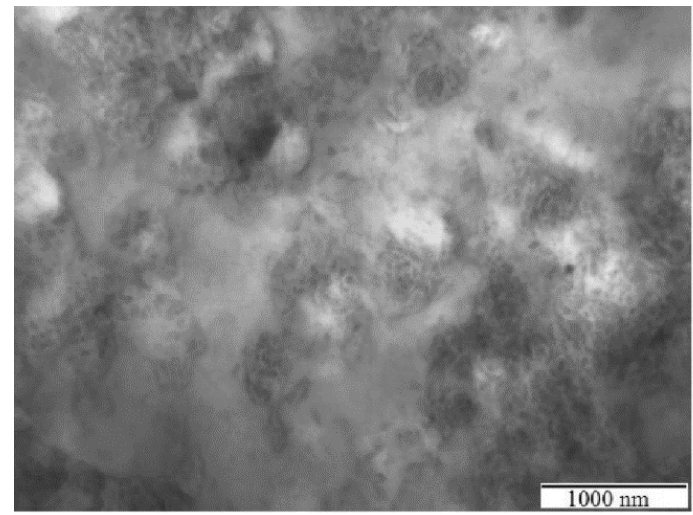

(a)

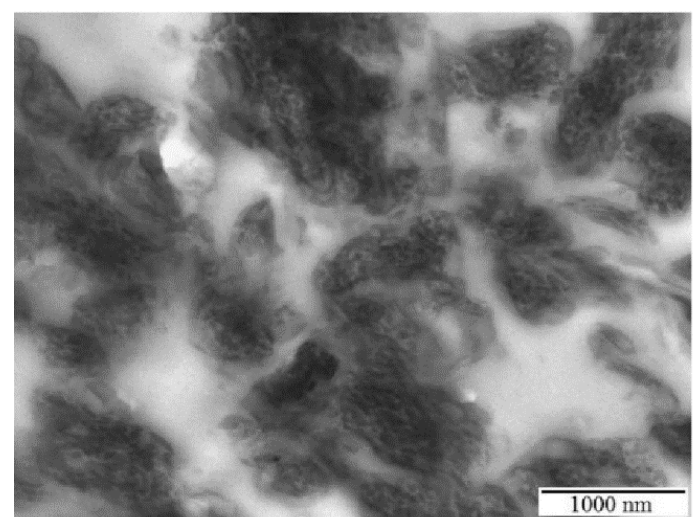

(c)

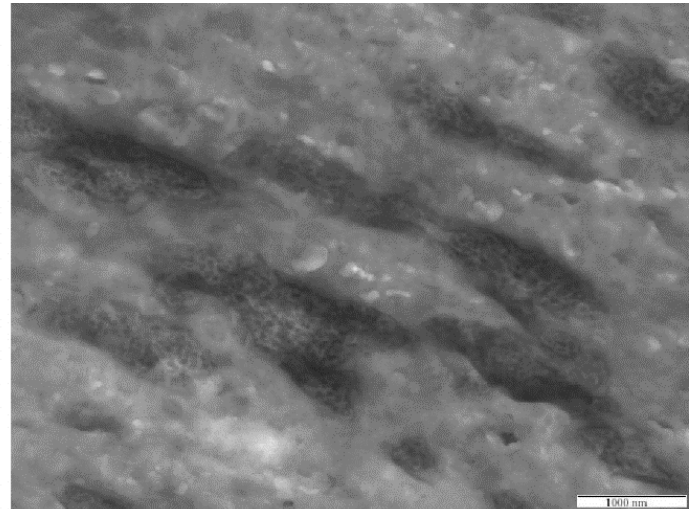

(b)

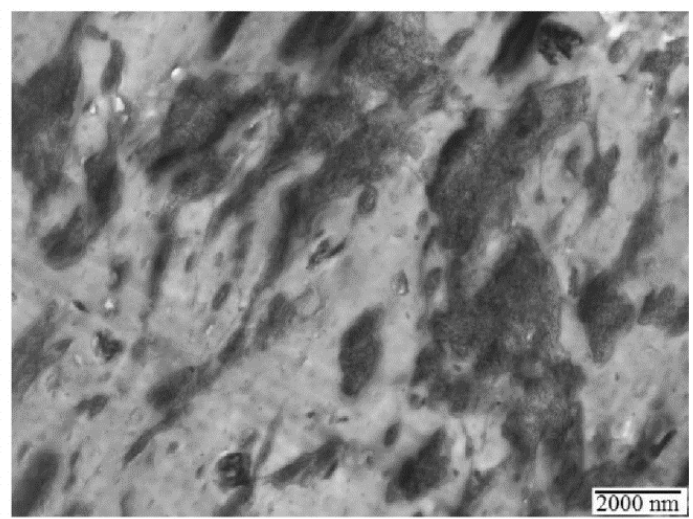

(d)

Figura 4. Micrografias obtidas por MET da blenda PA6/AES e dos nanocompósitos ternários PA6/AES/argila: (a) PA6/AES; (b) PA6/ AES/30B; (c) PA6/AES/20A e (d) PA6/AES/Na. 
que os sistemas com Cloisite ${ }^{\circledR 20 \mathrm{Ae} \mathrm{Cloisite}}{ }^{\circledR} \mathrm{Na}^{+}$apresentam estrutura intercalada e menor rigidez.

Observa-se também que a adição das nanocargas na blenda PA6/AES conduz a um aumento no módulo elástico e tensão no escoamento destes materiais; estas alterações são independentes da argila utilizada. No entanto, apesar deste aumento, os valores encontrados para os sistemas ternários (PA6/AES/argila) são significativamente menores que os da PA6 pura. Verifica-se ainda que assim como para os nanocompósitos binários uma redução na deformação na ruptura também foi observada para os sistemas ternários. Isto sugere que a blenda torna-se frágil na presença da nanoargila. A blenda PA6/AES sem argila possui deformação na ruptura de $38 \%$; a adição da Cloisite ${ }^{\circledR} 30 \mathrm{~B}$, Cloisite ${ }^{\circledR} 20 \mathrm{~A}$ e Cloisite ${ }^{\circledR} \mathrm{Na}^{+}$na blenda diminui a deformação para 15 , 7 e $18 \%$, respectivamente. Este comportamento pode ser devido a presença de tensões interfaciais, responsável pela transferência de tensões da matriz para a fase dispersa, que facilita a ruptura do material durante a solicitação.

A Figura 5 mostra os resultados obtidos através do ensaio de resistência ao impacto Izod com entalhe para a blenda PA6/AES e para os nanocompósitos binários e ternários obtidos com as diferentes nanocargas. Os valores destas propriedades e o desvio padrão encontram-se na Tabela 2.

Analisando os gráficos apresentados na Figura 5 é possível observar um aumento da resistência ao impacto dos nanocompósitos binários em relação a PA6 pura. Para os sistemas ternários a tenacidade é aumentada apenas para a mistura com Cloisite ${ }^{\circledR} \mathrm{Na}^{+}$, enquanto que para as blendas com Cloisite ${ }^{\circledR} 30 \mathrm{~B}$ e Cloisite ${ }^{\circledR} 20 \mathrm{~A}$ observa-se uma redução desta propriedade, quando se compara estas composições com a blenda PA6/AES. Este comportamento provavelmente pode está relacionado com as diferentes estruturas apresentadas por estes materiais. De acordo com Kusmonoet al. ${ }^{[6]}$ sistemas intercalados apresentam mais alta tenacidade que sistemas esfoliados. As lamelas de argila esfoliadas não são eficazes para prevenir a propagação da trinca. Adicionalmente, estes materiais rompem mais facilmente devido a sua maior rigidez. Consequentemente, os nanocompósitos PA6/AES/30B apresentam menor resistência ao impacto. Por outro lado, pode ocorrer cavitação nas vizinhanças dos tactóides de argila para os nanocompósitos PA6/AES/Na ${ }^{[6]}$. O mecanismo de cavitação pode ter favorecido a maior resistência ao impacto encontrada para os sistemas PA6/AES/Na em comparação com os nanocompósitos Cloisite ${ }^{\circledR}$ 30 e Cloisite ${ }^{\circledR} 20 \mathrm{~A}$.

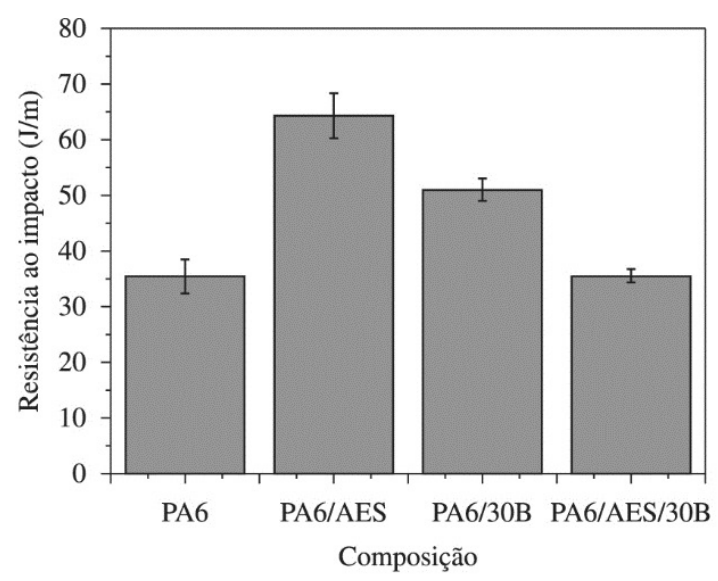

(a)

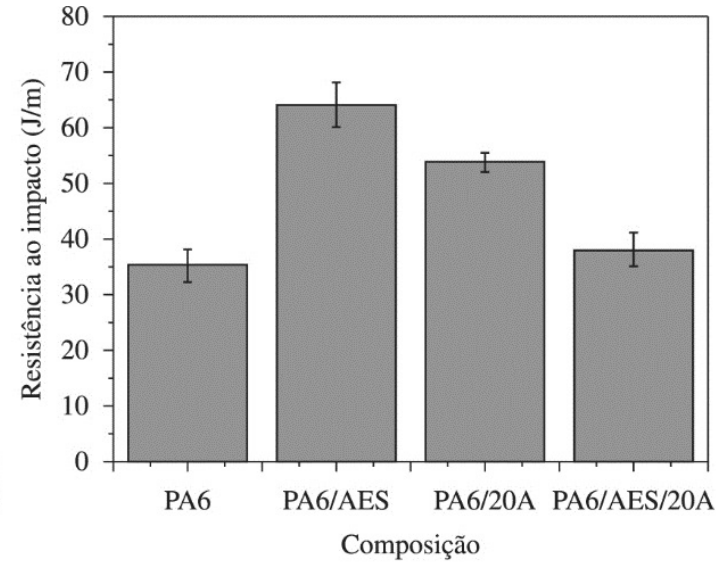

(b)

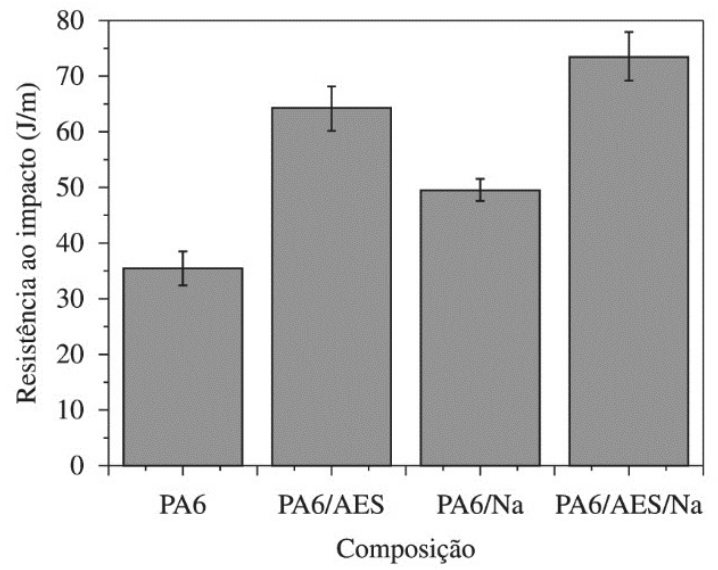

(c)

Figura 5. Resistência ao impacto Izod com entalhe da PA6 pura, da blenda PA6/AES nanocompósitos estudados: (a) sistemas com Cloisite ${ }^{\circledR} 30 \mathrm{~B}$; (b) sistemas com Cloisite ${ }^{\circledR} 2 \mathrm{~A}$; (c) sistemas com Cloisite ${ }^{\circledR} \mathrm{Na}^{+}$. 


\subsection{Caracterização termo-mecânica}

As curvas de E' e tan $\delta$ em função da temperatura, avaliadas em relação ao tipo de argila, são apresentadas nas Figuras 6 e 7, respectivamente. Os valores correspondentes a $\tan \delta$ encontram-se na Tabela 3.

O deslocamento do pico de $\tan \delta$ pode fornecer informações sobre as interações moleculares entre a PA6 e as nanoargilas. Observa-se na Figura 6a que a incorporação da Cloisite ${ }^{\circledR} 30 \mathrm{~B}$ não afeta significativamente a $\mathrm{Tg}$ da matriz, por outro lado, houve uma redução da Tg da PA6 para os nanocompósitos PA6/20A e PA6/Na. Este comportamento provavelmente está relacionado com a presença de uma interface mais forte e alto grau de esfoliação das lamelas de Cloisite ${ }^{\circledR} 30 \mathrm{~B}$ na matriz de PA6 em relação aos nanocompósitos com Cloisite ${ }^{\circledR} 20 \mathrm{~A}$ e Cloisite ${ }^{\circledR} \mathrm{Na}^{+}$. Para os sistemas ternários contendo AES (PA6/AES/argila), existe a formação de um pico bem definido para temperaturas nas proximidades de $110^{\circ} \mathrm{C}$, que representa a Tg do SAN. Verifica-se também a existência de dois ombros nestes sistemas, um em torno de -41 a $-39^{\circ} \mathrm{C}$ e outro em $53-64^{\circ} \mathrm{C}$, que representam a $\mathrm{Tg}$ da fase rica em EPDM e a Tg da parte amorfa da PA6, respectivamente.

A influência da nanocarga no módulo de armazenamento da PA6 também foi estudada (Figura 7). Observa-se que na temperatura ambiente ocorre uma redução no módulo de armazenamento com a adição de argilomineral para todos os nanocompósitos binários em relação à PA6 pura. Isto pode ser devido a grande rigidez apresentada por esta resina, como também foi verificado pelos valores de módulo elástico obtidos através dos ensaios de tração. Porém, um aumento no valor de E' foi encontrado para os nanocompósitos PA6/30B e PA6/20A para temperaturas próximas à $\mathrm{Tg}$ da PA6, uma vez que nesta faixa de temperatura o efeito de reforço da argila torna-se maior

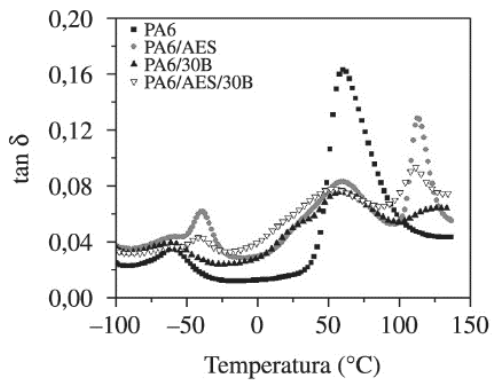

(a)

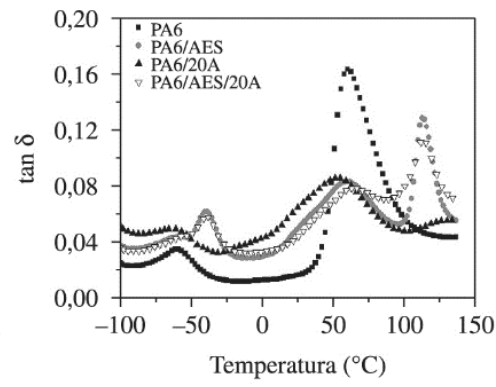

(b)

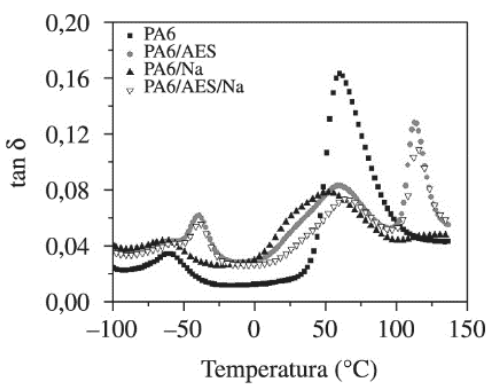

(c)

Figura 6. Curvas de tan $\delta$ em função da temperatura da PA6 pura, da blenda PA6/AES e dos nanocompósitos estudados: (a) sistemas com Cloisite ${ }^{\circledR} 30 \mathrm{~B}$; (b) sistemas com Cloisite ${ }^{\circledR} 20 \mathrm{~A}$; (c) sistemas com Cloisite ${ }^{\circledR} \mathrm{Na}^{+}$.

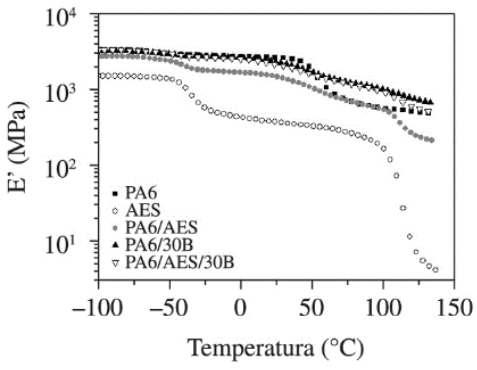

(a)

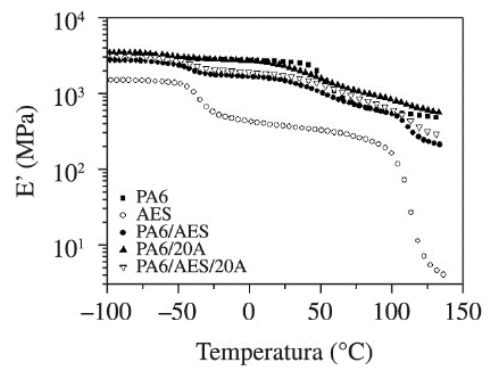

(b)

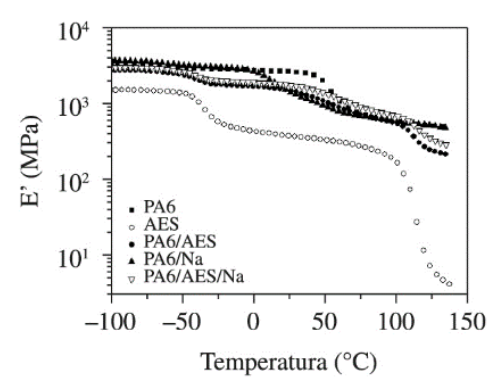

(c)

Figura 7. Módulo de armazenamento em função da temperatura dos sistemas estudados: (a) sistemas com Cloisite ${ }^{\circledR} 30 \mathrm{~B}$; (b) sistemas com Cloisite ${ }^{\circledR} 20 \mathrm{~A}$; (c) sistemas com Cloisite ${ }^{\circledR} \mathrm{Na}^{+}$.

Tabela 3. Propriedades termo-mecânicas das composições estudadas.

\begin{tabular}{|c|c|c|c|c|c|}
\hline \multirow{2}{*}{ Amostra } & \multirow{2}{*}{$\begin{array}{c}\mathrm{Tg}\left({ }^{\circ} \mathrm{C}\right) \\
\text { Fase rica em EPDM }\end{array}$} & \multirow{2}{*}{$\begin{array}{c}\operatorname{Tg}\left({ }^{\circ} \mathrm{C}\right) \\
\text { Fase rica em SAN }\end{array}$} & \multicolumn{2}{|c|}{ Tg (PA6) } & \multirow{2}{*}{$\mathrm{HDT}\left({ }^{\circ} \mathrm{C}\right)$} \\
\hline & & & Fase $\beta$ & Fase $\alpha$ & \\
\hline PA6 & ---- & ----- & $-59,6$ & 60,3 & $53,0 \pm 0,3$ \\
\hline AES & $-34,0$ & 115,2 & ----- & ------ & $65,7 \pm 2,0$ \\
\hline PA6/AES & $-39,5$ & 113,2 & ----- & 59,7 & $60,0 \pm 1,6$ \\
\hline PA6/30B & ----- & ----- & $-62,7$ & 58,9 & $87,5 \pm 7,0$ \\
\hline PA6/AES/30B & $-41,1$ & 110,9 & ----- & 53,0 & $80,0 \pm 7,0$ \\
\hline PA6/20A & ----- & ----- & $-64,1$ & 54,7 & $60,0 \pm 1,0$ \\
\hline PA6/AES/20A & $-39,0$ & 113,4 & ----- & 64,8 & $55,0 \pm 1,4$ \\
\hline $\mathrm{PA} 6 / \mathrm{Na}$ & ----- & ----- & $-62,6$ & 51,5 & $66,0 \pm 4,0$ \\
\hline PA6/AES/Na & $-39,0$ & 114,4 & ----- & 64,2 & $62,0 \pm 2,4$ \\
\hline
\end{tabular}


devido à redução do módulo da PA6 quando ela passa do estado vítreo para o borrachoso. A argila, no entanto, mantém a sua rigidez nesta mudança de temperatura. Comparando-se os nanocompósitos PA6/argila, observa-se que maiores aumentos de E' foram obtidos para as misturas PA6/30B, como esperado, dada à boa interface formada nesses nanocompósitos. Para os nanocompósitos PA6/Na, a presença de grandes tactóides de argila e baixa interação polímero-carga impossibilitou que o reforço mecânico fosse mais efetivo nestes materiais. Com relação aos sistemas PA6/AES/argila, pode-se observar um aumento significativo no valor de E' para todos os nanocompósitos em relação à blenda PA6/AES.

A Tabela 3 apresenta os resultados obtidos para a temperatura de deflexão térmica (HDT) dos sistemas estudados. A adição das nanoargilas aumenta a HDT da PA6, como esperado, sendo este efeito mais pronunciado para o nanocompósito PA6/30B, que foi de $65 \%$ em relação à matriz. Um aumento bastante significativo desta propriedade também foi observado pela adição da Cloisite ${ }^{\circledR 30 B}$ na blenda PA6/AES. A melhora apresentada pelos nanocompósitos com Cloisite ${ }^{\circledR} 30 \mathrm{~B}$ está relacionada com a melhor dispersão das lamelas deste silicato na matriz polimérica em relação às outras nanoargilas. Analisando os sistema ternários com Cloisite ${ }^{\circledR} 20 \mathrm{~A}$ e Cloisite ${ }^{\circledR} \mathrm{Na}^{+}$, verifica-se que ocorre uma redução da HDT para o sistema PA6/AES/20A em relação à blenda PA6/AES, e um ligeiro aumento desta propriedade para a mistura PA6/AES/Na.

\section{Conclusão}

Neste trabalho, nanocompósitos de blendas de PA6/AES foram obtidos através da adição de diferentes argilas montmorilonitas. Os resultados de WAXD e as micrografias obtidas por MET mostraram que a modificação da nanoargila apresenta grande influência na morfologia destes materiais. Para os nanocompósitos preparados com Cloisite ${ }^{\circledR}$ 30B observou-se um aumento do espaçamento basal desta nanocarga e nanocompósitos esfoliados foram obtidos. Por outro lado, as misturas com Cloisite ${ }^{\circledR} 20 \mathrm{~A}$ apresentaram estrutura intercalada e para todas as misturas com Cloisite ${ }^{\circledR} \mathrm{Na}^{+}$, verificou-se a presença de pequenos aglomerados de nanoargila. Os resultados de propriedades mecânicas mostraram um aumento mais significativo do módulo de elasticidade e tensão no escoamento para os sistemas com Cloisite ${ }^{\circledR} 30 \mathrm{~B}$. Já a Cloisite ${ }^{\circledR} \mathrm{Na}^{+}$foi mais eficaz em aumentar a tenacidade das misturas.

As análises termo-mecânicas mostraram que todas as nanocargas aumentam o módulo de armazenamento e a HDT da blenda, sendo este efeito mais pronunciado para as misturas ternárias com Cloisite ${ }^{\circledR} 30 \mathrm{~B}$. Isto foi atribuído à melhor dispersão desta nanocarga na matriz polimérica bem como à melhor interação da Cloisite ${ }^{\circledR} 30 \mathrm{~B}$ com a PA6.

\section{Agradecimentos}

Os autores agradecem ao CNPq pelo apoio financeiro e a petroquímica Polyform pela doação da poliamida 6 .

\section{Referências}

1. Paul, D. R., \& Robeson, L. M. (2008). Polymer nanotechnology: nanocomposites. Polymer, 49(15), 3187-3204. http://dx.doi. org/10.1016/j.polymer.2008.04.017.

2. Brito, G. F., Oliveira, A. D., Araújo, E. M., Melo, T. J. A., Barbosa, R., \& Ito, E. N. (2008). Nanocompósitos de polietileno/ argila bentonita nacional: influência da argila e do agente compatibilizante PE-g-MA nas propriedades mecânicas e de inflamabilidade. Polímeros Ciência e Tecnologia, 18(2), 170177. http://dx.doi.org/10.1590/S0104-14282008000200015.

3. Oliveira, A. D., Larocca, N. M., Paul, D. R., \& Pessan, L. A. (2012). Effects of mixing protocol on the performance of nanocomposites based on polyamide 6/acrylonitrile-butadienestyrene blends. Polymer Engineering and Science, 52(9), 1909-1919. http://dx.doi.org/10.1002/pen.23152.

4. Li, Y., \& Shimizu, H. (2005). Co-continuous Polyamide 6 (PA6)/Acrylonitrile-Butadiene-Styrene (ABS) nanocomposites. Macromolecular Rapid Communications, 26(9), 710-715. http://dx.doi.org/10.1002/marc.200400654.

5. Lan, T., \& Pinnavaia, T. J. (1994). Clay-reinforced epoxy nanocomposites. Chemistry of Materials, 6(12), 2216-2219. http://dx.doi.org/10.1021/cm00048a006.

6. Kusmono, Z. A., Ishak, Z. A. M., Chow, W. S., \& Rochmadi, T. T. (2010). Effect of clay modification on the morphological, mechanical, and thermal properties of polyamide 6/polypropylene/ montmorillonite nanocomposites. Polymer Composites, 31(7), 1156-1167. http://dx.doi.org/10.1002/pc.20902.

7. Dennis, H. R., Hunter, D. L., Chang, D., Kim, S., White, J. L., Cho, J. W., \& Paul, D. R. (2001). Effect of melt processing conditions on the extent of exfoliation in organoclay-based nanocomposites. Polymer, 42(23), 9513-9522. http://dx.doi. org/10.1016/S0032-3861(01)00473-6.

8. Gallego, R., García-López, D., Merino, J. C., \& Pastor, J. M. (2010). The effect of montmorillonite and compatibilizer quantities on stiffness and toughness of polyamide nanoblends. Polymer International, 59(4), 472-478. http://dx.doi.org/10.1002/pi.2724.

9. Oliveira, A. D., Larocca, N. M., \& Pessan, L. A. (2011). Efeito da sequência de mistura nas propriedades de blendas PA6/ ABS compatibilizadas com o copolímero SMA. Polímeros: Ciência e Tecnologia, 21(1), 27-33. http://dx.doi.org/10.1590/ S0104-14282011005000010.

10. Araújo, E. M., Hage, J. E., \& Carvalho, A. J. F. (2004). Compatibilização de blendas de poliamida 6/ABS usando os copolímeros acrílicos reativos MMA-GMA e MMA-MA. Parte 2: comportamento termomecânico e morfológico das blendas. Polímeros: Ciência e Tecnologia, 14(1), 22-30. http://dx.doi. org/10.1590/S0104-14282004000100010.

11. Wang, C., Su, J. X., Li, J., Yang, H., Zhang, Q., Du, R. N., \& Fu, Q. (2006). Phase morphology and toughening mechanism of polyamide 6/EPDM-g-MA blends obtained via dynamic packing injection molding. Polymer, 47(9), 3197-3206. http:// dx.doi.org/10.1016/j.polymer.2006.03.012.

12. Bassani, A., Hage, J. E., \& Pessan, L. A. (2002). Propriedades mecânicas de blendas de nylon-6/Acrilonitrila-EPDM-Estireno (AES) compatibilizadas com copolímero acrílico reativo (MMA-MA). Polímeros: Ciência e Tecnologia, 12(2), 102108. http://dx.doi.org/10.1590/S0104-14282002000200009.

13. Dijkstra, K., Ter Laak, J., \& Gaymans, R. J. (1994). Nylon-6/ rubber blends: 6 . Notched tensile impact testing of nylon-6/ (ethylene-propylene rubber) blends. Polymer, 35(2), 315-322. http://dx.doi.org/10.1016/0032-3861(94)90696-3.

14. Covas, J. A., Machado, A. V., Pessan, L. A., Hage, E., \& Bassani, A. (2005). Evolução da morfologia de fases de blendas PA6/ AES em extrusora de dupla rosca e moldagem por injeção. 
Polímeros Ciência e Tecnologia, 15(3), 176-185. http://dx.doi. org/10.1590/S0104-14282005000300007.

15. Zhang, L., Wan, C., \& Zhang, Y. (2008). Polyamide 6/maleated ethylene-propylene-diene rubber/organoclay composites with or without glycidyl methacrylate as a compatibilizer. Journal of Applied Polymer Science, 110(3), 1870-1879. http://dx.doi. org/10.1002/app.28768.

16. Dasari, A., Yu, Z. Z., \& Mai, Y. W. (2005). Effect of blending sequence on microstructure of ternary nanocomposites. Polymer, 46(16), 5986-5991. http://dx.doi.org/10.1016/j. polymer.2005.05.145.

17. Lu, M., Keskkula, H., \& Paul, D. R. (1995). Toughening of nylon 6 with grafted rubber impact modifiers. Journal of Applied Polymer Science, 58(7), 1175-1188. http://dx.doi. org/10.1002/app.1995.070580711.

18. Larocca, N. M., Hage, J. E., Jr, \& Pessan, L. A. (2004). Toughening of poly (butylene terephthalate) by AES terpolymer.
Polymer, 45(15), 5265-5277. http://dx.doi.org/10.1016/j. polymer.2004.05.056.

19. Passador, F. R., Backes, E. H., Travain, D. R., Filho, A. R., \& Pessan, L. A. (2013). Nanocompósitos de blendas HDPE/ LLDPE e OMMT - Parte I: avaliação das propriedades termomecânicas e da resistência ao intemperismo. Polímeros: Ciência e Tecnologia, 23(4), 521-530. http://dx.doi.org/10.4322/ polimeros.2013.056.

20. Martins, C. G., Larocca, N. M., Paul, D. R., \& Pessan, L. A. (2009). Nanocomposites formed from polypropylene/EVA blends. Polymer, 50(7), 1743-1754. http://dx.doi.org/10.1016/j. polymer.2009.01.059.

Enviado: Jun. 17, 2014 Revisado: Nov. 18, 2014 Aceito: Jan. 07, 2015 